\title{
Abelian and non-abelian symmetries in infinite projected entangled pair states
}

\author{
Claudius Hubig \\ Max-Planck-Institut für Quantenoptik, \\ Hans-Kopfermann-Str. 1, 85748 Garching, Germany \\ claudius.hubig@mpq.mpg.de
}

21st July 2021

\begin{abstract}
We show how to implement arbitrary abelian and non-abelian symmetries in the setting of infinite projected entangled pair states on the two-dimensional square lattice. We observe a large computational speed-up obtained through the increased sparsity and reduced tensor sizes; easily allowing bond dimensions $D=10$ in the square lattice Heisenberg model at computational effort comparable to calculations at $D=6$ without symmetries. We also find that absent spontaneous symmetry breaking, implementing a symmetry does not negatively affect the representative power of the state and leads to identical ground-state energies. Furthermore, we point out how we can use symmetry implementations to detect such spontaneous symmetry breaking.
\end{abstract}

\section{Introduction}

Tensor network methods have become the de facto method of choice in the numerical treatment of one-dimensional quantum systems [1]. The extension to other loop-free geometries is relatively straightforward [2 7] and preserves most of the numerical advantages of the onedimensional systems. Work is still ongoing, however, to effectively and efficiently handle large two-dimensional systems which are firstly both of extremely high experimental and theoretical interest and secondly difficult to treat numerically with any method. For example, the dynamical mean-field theory is being combined with new and different solvers $[7,9]$ to allow the treatment of larger clusters within the dynamical cluster approximation; a similar approach is taken for the density matrix embedding theory [10,11 to likewise allow for larger clusters [12] potentially necessary for the treatment of two-dimensional lattices. Recently, a diagrammatic formulation of quantum Monte Carlo [13] was also used to further avoid the sign problem, which otherwise makes calculations in frustrated or fermionic systems very hard. Standard matrix-product state/density-matrix renormalisation group (MPS-DMRG) methods are also continuously applied to wider and wider cylinders, but computational costs essentially explode beyond relatively limited cylinder circumferences 14 17.

Projected entangled pair states [18], the two-dimensional generalisation of matrix-product states, were proposed soon after the understanding of the latter in the context of the density matrix renormalisation group and a further generalisation to infinite two-dimensional systems [19,20] followed with continuous improvements $[21,29]$ to the method over the last few 
years. In this work, we will combine the exact implementation of both abelian and non-abelian symmetries - which is a standard method in one-dimensional MPS codes - with the infinite projected entangled pair states (IPEPS) method used to calculate two-dimensional groundstate properties. In particular, we will show that, absent spontaneous symmetry breaking, implementing an abelian or non-abelian symmetry provides a major computational advantage and considerably improved estimates for the ground-state energy. This will be done using the explicit Clebsch-Gordan Coefficients approach presented in Ref. [30]. Furthermore, we will showcase how the implementation of a symmetry allows additional control over a calculation: First, once the symmetry is implemented, one forces the calculation into a canonical ensemble, hence exactly preserving e.g. particle number at the desired and manually-selected value. Second, enabling or disabling the preservation of the symmetry allows selecting between symmetry-unbroken and symmetry-broken states and phases. By comparing the energies of these states, it becomes very easy and very stable to pinpoint phase transitions.

To this end, the paper is structured as follows: Sec. 2 reviews the implementation of arbitrary symmetries in tensor networks and establishes some notation. Sec. 3 goes into further detail of required adaptations of the standard IPEPS algorithms to allow for such an implementation as well as different considerations of computational effort. In Sec. 4, we use the Heisenberg $S=1 / 2$ Hamiltonian on a square and Kagomé lattice to show the computational speed-up attainable by implementing symmetries in the network. In particular, a large benefit due to the implementation of the unbroken $\mathrm{SU}(2)-S$ symmetry in the Kagomé lattice is observed. The selection of symmetry-broken and symmetry-unbroken states and their use in diagnosing spontaneous symmetry breaking is discussed in Sec. 5 . Finally, we conclude in Sec. 6.

\section{Symmetries in tensor networks}

The implementation of global abelian symmetries in low-rank loop-free tensor networks is generally well-understood and a cornerstone of efficient computations in modern codes 1 , 31 33. In contrast to this, global non-abelian symmetries 34 have seen less widespread use and proposals on how to implement them for higher-rank tensor networks are relatively new [30,35]. While global abelian symmetries generalise straightforwardly to higher-rank tensors (see e.g. Ref. [36] for an application to PEPS), for non-abelian symmetries there are two alternative approaches: the implicit Clebsch-Gordan coefficients approach relies on analytical knowledge of Clebsch-Gordan coefficients (CGCs) for rank-3 tensors and decomposes any higher-rank tensor into a double tree of rank-3 tensors [35, 37]. Alternatively, the explicit $C G C$ approach generalises the notion of CGCs to higher-rank tensors and explicitly stores those coefficients within the tensor [30]. The latter approach was already applied to IPEPS calculations to some extent in Ref. [38].

Here, we will briefly summarise the implementation of arbitrary symmetries following the explicit CGC approach as done in the SyTEN toolkit [39]. For a more extensive discussion of this approach, see Refs. [30,40]. In Sec. 3 we will then go on to discuss implementation details specific to IPEPS calculations.

Fundamentally, a rank- $R$ tensor $T$ is represented as a direct sum of a finite number of 
blocks $\left\{t_{i}\right\}$ :

$$
T=\bigoplus_{i=1}^{N_{t}} t_{i}
$$

Each of the $R$ legs of the tensor is given a direction corresponding to the tensor acting on the vector space on this leg or its dual. A matrix, for example, would have one incoming leg and one outgoing leg. Every block $t_{i}$ then firstly contains a single quantum number label for every symmetry in the system (e.g. U(1) particle number $n$ and $\mathrm{SU}(2)$ spin $S$ ) on each of its $R$ legs. Furthermore, each block contains one dense reduced rank- $R$ tensor $m_{i}$ and, for every symmetry $\gamma$, a generalised $C G C$ rank- $R$ tensor $c_{i}^{\gamma}$ :

$$
t_{i}=m_{i} \bigotimes_{\gamma=1}^{N_{S}} c_{i}^{\gamma}
$$

Blocks with the same quantum numbers on all legs can be added directly, blocks with different quantum numbers are placed next to each other. If there are no symmetries in the system, the tensor $T$ only contains a single block $t_{1}$ which, in turn, only contains the collection of scalar coefficients of the tensor. For abelian symmetries, $c_{i}^{\gamma}$ are one-dimensional tensors which are non-zero if the addition rules for the abelian symmetry are fulfilled by the quantum numbers assigned to the tensor legs and zero if the addition rules are broken. Alternatively, the $c_{i}^{\gamma}$ can be left off and the addition rules enforced by hand. Note that here we force every tensor to preserve the symmetry exactly and do not allow for a shift of all quantum numbers within a tensor, as such shifts are only well-defined for abelian symmetries and would break down immediately when including non-abelian symmetries. In these latter cases, the $c_{i}^{\gamma}$ are generally non-trivial; for rank-2 tensors, they must, however, be proportional to identity matrices and for rank-3 tensors, they correspond directly to the standard Clebsch-Gordan coefficients.

Tensor operations can then be implemented to largely act independently on each block and also the $m_{i}$ and $c_{i}^{\gamma}$ within each block. The addition of two tensors $T+S$ is simply a direct sum over all blocks:

$$
T+S=\bigoplus_{x=t, s} \bigoplus_{i=1}^{N_{x}} x_{i} .
$$

However, we may then end up with many blocks with identical quantum numbers on all legs and an identical Clebsch-Gordan structure. If for every symmetry in the system, the associated CGC tensors of two blocks are parallel to each other, we may move those factors into the reduced dense tensors and then add the reduced dense tensors together to arrive at a single block consisting of the sum of the dense tensors as well as one set of CGC tensors. This operation hence reduces the number of blocks in the direct sum and is called a reduction in the following (not to be confused with the reduced tensor $m_{i}$ above).

The contraction of two tensors $T$ and $S, R=T \cdot S$ over a set of legs is evaluated as follows: First, collect all pairs of blocks $\left\{t_{i}, s_{j}\right\}$ which are labelled by identical quantum numbers on the contracted legs. Then construct result blocks $r_{k}$ as

$$
r_{k}=\left(m_{t, i} \cdot m_{s, j}\right) \bigotimes_{\gamma=1}^{N_{s}}\left(c_{t, i}^{\gamma} \cdot c_{s, j}^{\gamma}\right)
$$

where denotes the desired contraction and afterwards reduce the number of blocks of the result tensor $R$ as described above by adding parallel tensor blocks. 
While it is straightforward to parallelise over the pairs of all possible contractions, to reduce memory usage, one should also first collect all contraction pairs resulting in a given quantum number configuration on the open legs, contract and reduce those and only then move on to the next set of quantum numbers on the open legs. Otherwise, the intermediate, unreduced $R$ tensor can easily exceed the size of the actual reduced $R$ by a factor of 100 .

Reduced and Total Dimensions When speaking about the bond dimension of a tensor network state or more generally the dimensions of a tensor, it is useful to differentiate between the reduced and the total dimension. Here, the reduced dimension of a tensor on one of its legs is the sum of the sizes of every reduced block (counted only once per quantum number sector). In comparison, the total dimension of a tensor on one of its legs is the sum of the sizes of every reduced block multiplied by the product of the sizes of the associated generalised CGC tensors. Put another way, a spin doublet state with two duplicate singular values associated to $\pm 1 / 2$ has a total dimension of two regardless of the symmetries used to represent it. However, if the tensor implements the $\mathrm{SU}(2)$ spin symmetry, the reduced dimension of the state is merely 1 , the duplicate-ness of the singular values is not counted. We then typically expect computational effort to scale cubically in the reduced dimensions, whereas the expressivity of a tensor and the potentially carried entanglement scales with its total dimensions. To differentiate the two in the following text, we will use capital letters $(D, X)$ to refer to total dimensions whereas lower-case letters $(d, \chi)$ refer to the reduced dimensions. In the IPEPS calculations to follow, $d$ and $D$ respectively will denote the state bond dimension whereas $\chi$ and $X$ respectively will denote the environment bond dimension.

The distribution of states among the different quantum number sectors is in principle open and should be adapted according to the specific state represented. While at relatively small bond dimensions (as encountered in IPEPS calculations) it may be feasible to fix that distribution by hand beforehand, finding the optimal choice a priori becomes combinatorially expensive. To avoid this problem, minimal care must be taken to allow flexibility in the choice of quantum number sectors. Within the full update scheme with imaginary time evolution, this flexibility comes naturally. For the newer variational update schemes [27, 28] one may have to consider either two-site updates or an expansion scheme analogous to the subspace expansion in DMRG 41.

\section{Implementation details specific to IPEPS}

Our IPEPS calculation largely follows the existing literature, making use of imaginary time evolution with the fast full update [25] and gauge fixing [24,26]. The corner transfer matrix is truncated as suggested in [23.

Errors for observables from the corner transfer matrix method are estimated as follows: First, at given $\chi$, CTM steps are done until the observable changes by less than a small threshold $\left(10^{-8}\right)$ or 15 steps are done. The first error is then the maximum change between the last three steps, the last value is taken as the result of this fixed- $\chi$ calculation. Second, $\chi$ is multiplied by 2 and the previous procedure repeated until the resulting values differ by less than the small threshold $10^{-8}$ or $\chi$ is larger than 128 . The second error is then the change between the value at the last fixed $\chi$ and the second-to-last calculation at fixed $\chi$. The error is the larger of the first and second error. In our experience, this is a very conservative error 


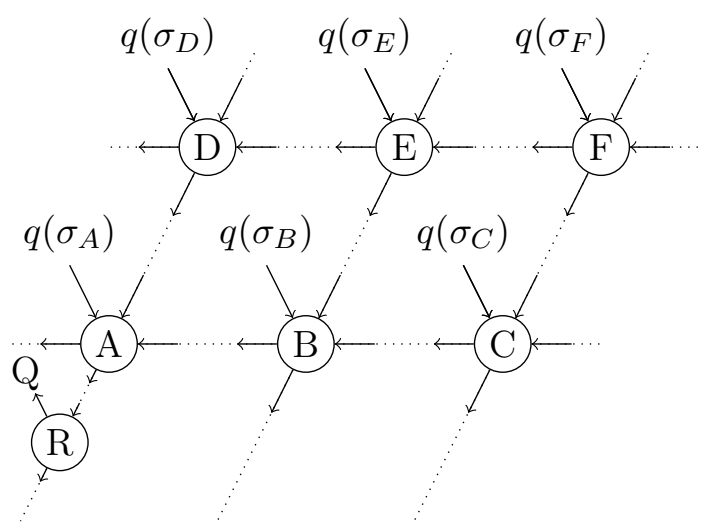

Figure 1: Layout of an example $2 \times 3$ IPEPS unit cell as used here. Each of the individual site tensors $A, B, C, D, E$ and $F$ inserts some charge into the network (which also depends on the local state), the special remover tensor $R$ is used to extract a total charge $Q$ from the unit cell. Auxiliary leg contractions are indicated by the dotted lines. The average quantum number on the inter-unit-cell auxiliary legs is zero (with some fluctuations) whereas intra-unit-cell legs can have non-zero average quantum number depending on the values of $q(\sigma)$ and the "routing" of charges through the unit cell.

measure which is likely to overestimate the error of the obtained value. Absent a better error estimation method, we will however use it in the following figures.

While the variational update is not yet implemented, its improvements are orthogonal to those discussed here, so we expect all results to carry over from imaginary time evolution to the variational update with minimal modifications. The same applies to the new extrapolation schemes for observables [29,42].

\subsection{Required adaptations of the IPEPS setup}

The first step to combine symmetry-preserving tensors with an established tensor network algorithm is to define a consistent leg direction such that the tensor contractions required to, for example, represent the state are well-defined. The specific leg directions are entirely arbitrary, but of course have to be consistent within a calculation 17

Second, when enforcing the symmetry, we effectively work in a canonical ensemble of a fixed average quantum number per unit cell. If this average quantum number is non-zero (e.g. 1 particle per site in the Hubbard model), it shifts the outgoing auxiliary tensor legs relative to the incoming auxiliary tensor legs. To account for this shift and give an overall consistent state, we insert an additional rank-3 remover tensor on the boundary of each unit cell. The remover tensor has a "physical" leg which points in the opposite direction of the usual physical legs on our IPEPS sites and simply removes as many particles as desired from the calculation. The additional leg is traced out during all calculations, for abelian symmetries, it amounts to an additional shift of the quantum numbers in each unit cell.

Third, when constructing a single-site operator such as $\hat{s}^{+}$in a U(1)- $S^{z}$ preserving system, it is necessary to insert an additional tensor leg to carry the additional quantum number. In

\footnotetext{
${ }^{1}$ Even when not enforcing the symmetry at the tensor level, a consistent choice of directions is required, as observed e.g. in Ref. 43.
} 
the product $\hat{s}_{i}^{+} \hat{s}_{j}^{-}$, these legs then combine to form a two-site gate acting on sites $i$ and $j$ connected by the contracted leg. If the contracted leg crosses any other tensor legs, the usual rule for fermionic PEPS apply [21] and a swap gate has to be inserted 22]. This swap gate fulfills the same role as Jordan-Wigner strings between operators $\hat{c}^{\dagger}$ and $\hat{c}$ in matrix-product operators 44 .

\subsection{Performance and accuracy necessitated by IPEPS compared to MPS}

In a typical MPS-DMRG calculation, the maximal ranks of temporary tensors is typically limited to four or five, with the basis state building blocks having rank three. This has two beneficial side effects: first, the number of blocks in a tensor is limited to approximately $O(1000)$ with each individual block being relatively large. Second, the entries of the CGC tensors are regularly constructed from rank-2 and rank-3 tensors, which helps alleviate the buildup of numerical errors due to limited accuracy.

In an IPEPS calculation, in contrast, the maximal tensor ranks are much larger (rank 10 or above!), naively leading to tensors with $O\left(10^{6}\right)$ or more blocks. Furthermore, numerical errors in the CGC tensors build up very quickly as the basic rank- 5 tensors already display a complicated Clebsch-Gordan structure.

The first issue is solely a performance problem and can be avoided by minimising tensor ranks wherever possible. In particular, it turns out that first constructing the rank-4 doublelayer tensors and then applying the CTM algorithm based on the rank-4 tensors is effectively computationally more efficient than contracting the single-layer rank-5 tensors individually into the existing CTM tensors, even though the latter approach is asymptotically cheaper. However, the former decreases the overhead both from bookkeeping the tensors and initialising many extremely small matrix-matrix products.

The second problem is more intricate and delayed by three tricks: First, we use higherprecision floating point numbers 2 to represent the entries of the CGC tensors. Second, within a tensor, we regularly check all non-zero coefficients (of which there are typically very few) and set those coefficients which are close in magnitude equal to each other. As an illustrative example, if a CGC tensor contains coefficients $[0.99,1.01,-1.01,0.99]$, we set them to $[1.00,1.00,-1.00,1.00]$. The actual closeness threshold used is $\approx 10^{-16}$. This relies on the fact that the coefficients of a CGC tensor are computed from very few inputs in very specific ways and no (typical) CGC tensor should truly contain coefficients different from each other but closer than $10^{-16}$. Finally, it is helpful to regularly reconstruct the CGC tensors from scratch to remove all numerically buildup errors. This is done by first merging all tensor legs of the tensor until one arrives at a tensor with a single incoming and a single outgoing leg. In this tensor, the CGC tensors should be proportional to identity matrices which is then ensured manually. Afterwards, the tensor legs are split again to rebuild the original tensor structure.

An alternative approach would be to use continued fractions to represent the CGC coefficients to high accuracy as suggested in Ref. [30,38] at the cost of a higher effort to implementation.

With these relatively minor changes, it becomes possible to do $\mathrm{SU}(2)$ - and $\mathrm{U}(1)$-symmetric calculations on infinite projected entangled pair states using symmetric tensors in place of standard dense tensors without internal structure.

\footnotetext{
${ }^{2}$ Specifically, the boost: :multiprecision: :cpp_bin_float $\langle 76>$ type is used.
} 


\section{Computational speed-up}

In classical DMRG calculations, implementing an abelian U(1) symmetry typically leads to an approximately ten-fold computational speed-up or, alternatively, an approximately tripled bond dimension. Changing from a U(1) to a SU(2) symmetry has a similar effect of approximately tripling the effective bond dimension. To test the effect of the implemented symmetries, we pick two example systems, namely $S=1 / 2$ spins on the Kagomé and square lattice.

\subsection{Spin-1/2 square lattice Heisenberg model}

As a first example, we consider the $S=1 / 2$ Heisenberg Hamiltonian on a square lattice at the Heisenberg point of isotropic coupling:

$$
\hat{H}=\sum_{\langle i, j\rangle} \hat{s}_{i}^{x} s_{j}^{x}+\hat{s}_{i}^{y} \hat{s}_{j}^{y}+\hat{s}_{i}^{z} \hat{s}_{j}^{z}
$$

The system spontaneously breaks the $\mathrm{SU}(2)$ symmetry of the Hamiltonian but preserves both the $\mathbb{Z}_{2}$ and $\mathrm{U}(1)-S^{z}$ symmetries.

The computational speed-up obtainable is first estimated by measuring the time required to re-initialise the corner transfer matrix with eight steps in each direction, evaluate the energy (evaluating eight two-site expectation values) and then do five full updates for imaginary time evolution of a second-order Trotter decomposition on a 2x2 unit cell. Each step requires the application of 16 gates. 8 of the 16 gates connect two neighbouring unit cells and hence also require a directional (up/down or left/right) CTM step. The runtime measurement is done after sufficiently many initial steps that the IPEPS bond dimension $d$ and the bond dimension $\chi$ of the environment has saturated. For abelian (or no) symmetries, we set $\chi=10(d+1)$, while for the non-abelian $\mathrm{SU}(2)$ case, $\chi=5(d+1)$ appears to be enough to obtain stability and convergence.

Fig. 2 shows the CPU time in seconds for the above procedure. These measurements were done on a single core of a Intel Xeon CPU E5-2630 v4 clocked at 2.20GHz. Except at minimal bond dimension $D=2$, enforcing the $\mathrm{U}(1)$ or $\mathbb{Z}_{2}$ symmetry has a very beneficial effect on the runtime. At larger bond dimensions, the U(1)-symmetric calculation is also much faster than the $\mathbb{Z}_{2}$-symmetric calculation. Comparing the reduced bond dimension $d$ of a $\mathrm{SU}(2)$-symmetric calculation, one observes a very large cost (e.g. at $d=3: 89,22,12$ and 653 seconds respectively without symmetries, with $\mathbb{Z}_{2}, \mathrm{U}(1)$ and $\left.\mathrm{SU}(2)\right)$. Part of this large cost is due to a relatively large overhead of the CGC tensors, but another part comes from intermediate tensors (e.g. the rank-4 double layer tensors) having a larger relative bond dimension which is less amenable to a reduction in size by the symmetry implementation. On the other hand, when comparing the total effective bond dimension $D$, the $\mathrm{SU}(2)$-symmetric implementation is always faster (at $D=4,8,12$ ) than a comparable calculation without this symmetry. Due to the model spontaneously breaking the SU(2) symmetry in the ground state the obtained energies are not comparable and the data is included here only for completeness.

The error in energy per spin with and without symmetries is given in Fig. 3 relative to the reference value $E_{0} \equiv-0.669437[45]$. The calculation is initialised in a Néel state $(D=1)$ or (for the $\mathrm{SU}(2)$ case) two singlets $(D=2)$ and then proceeds in stages. We first increase 


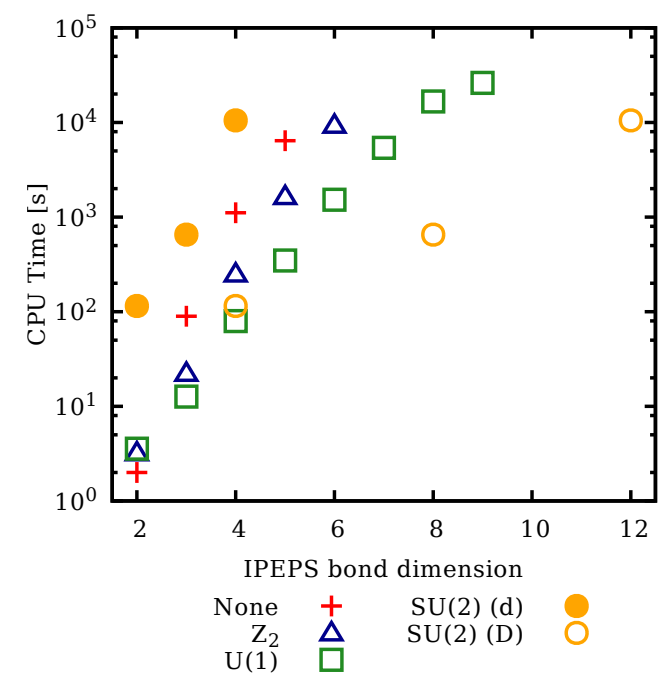

Figure 2: Computational effort for CTM initialisation, energy evaluation and five FFU steps in imaginary time in the Heisenberg square lattice model. Data for the SU(2)symmetric calculation is plotted over the reduced $(d)$ and total $(D)$ bond dimension.

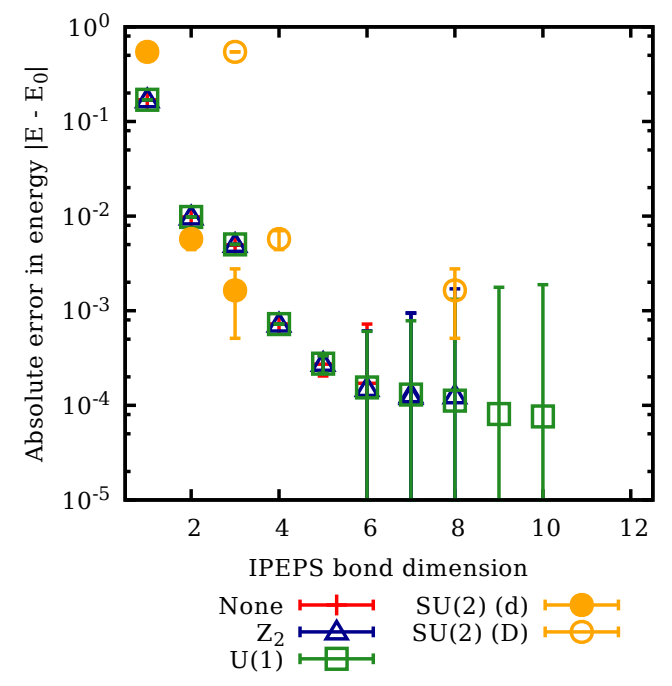

Figure 3: Error in energy per spin calculated in the Heisenberg square lattice model at $\Delta=1$ with $E_{0}=-0.669437$. Error bars are obtained from the CTM calculation and give the error of the observable relative to its true value in the given variational state.

the bond dimension to $d=2$ and then time evolve with step size $\delta \tau=1,0.1,0.01$ until the energy (estimated every five steps) stops decreasing. Subsequently, we either decrease $\delta \tau$ or, if we reached the minimal specified $\delta \tau$, increase $d$ and reset $\delta \tau$ to a larger value (0.1). Starting at $d=3$, we allow a smaller minimal $\delta \tau=0.001$. The Trotter error should hence be of order $O\left(\delta \tau^{2}\right) \approx 10^{-6}$. The energy estimates during the time evolution are at a fixed $\chi$ and with a re-initialised environment to trade-off accuracy and computational effort. Each obtained state is however also saved to disk.

The expectation values shown are then evaluated on the stored states as described in Sec. 3 , in particular, error bars estimate the error of the calculated value compared to the true value of the given state. Due to our maximal environment bond dimension being limited to $\chi=128$, error bars become relatively large in the logarithmic plot. Data for $\mathrm{SU}(2)$ is only included for completeness, so let us focus on the three other curves for calculations with no symmetries, $\mathbb{Z}_{2}$ symmetry and $\mathrm{U}(1)$ symmetry:

Contrary to Ref. [36 we find very comparable results with and without symmetries at identical bond dimensions. In particular at larger bond dimensions $(D=5,6)$, enforcing the symmetry appears to aid the calculation as we can guide the state into the correct sector. This difference is most likely an effect of the fixed quantum number distribution with an equal number of states per sector used in Ref. [36] which reduces the expressive power of the state.

As expected, growing computational effort makes calculations at larger $D$ only accessible with the full $\mathrm{U}(1)$ symmetry. In addition to the reduced matrix sizes, the blocking of tensors also allows for a straightforward shared-memory parallelisation which was used here to obtain converged $D \geq 7$ results at acceptable wallclock times of no more than two days. For these larger bond dimensions, we observe that the obtained error gradually ceases to shrink. We expect that a variational updates of the tensors should decrease the energy further. 


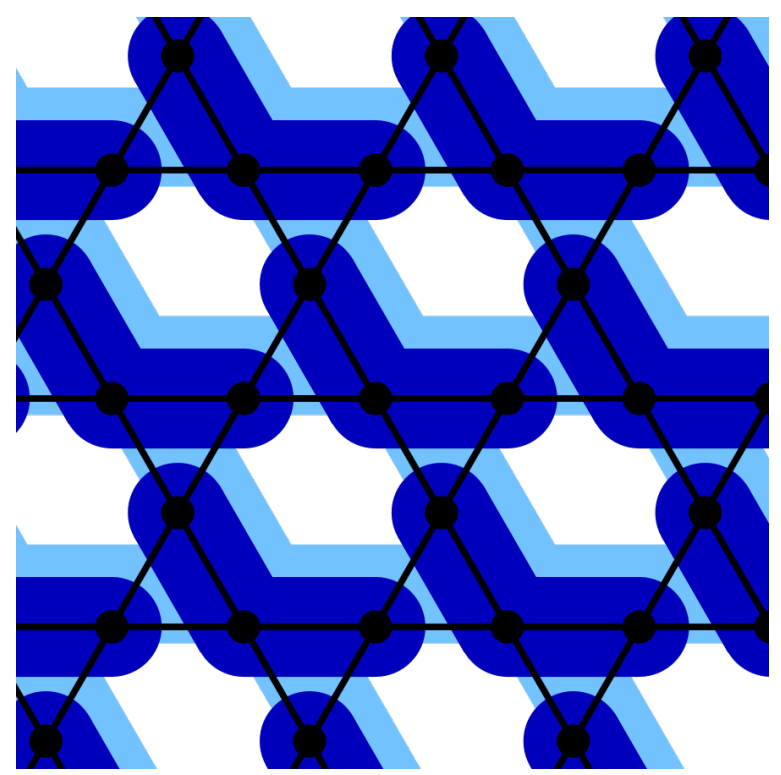

Figure 4: The mapping of the Kagomé lattice to the square lattice used here. Three sites of the Kagomé lattice (black circles) are merged into one site of the square lattice (dark blue). Each link between two square-lattice sites (light blue) corresponds to two links of the Kagomé lattice (black lines).

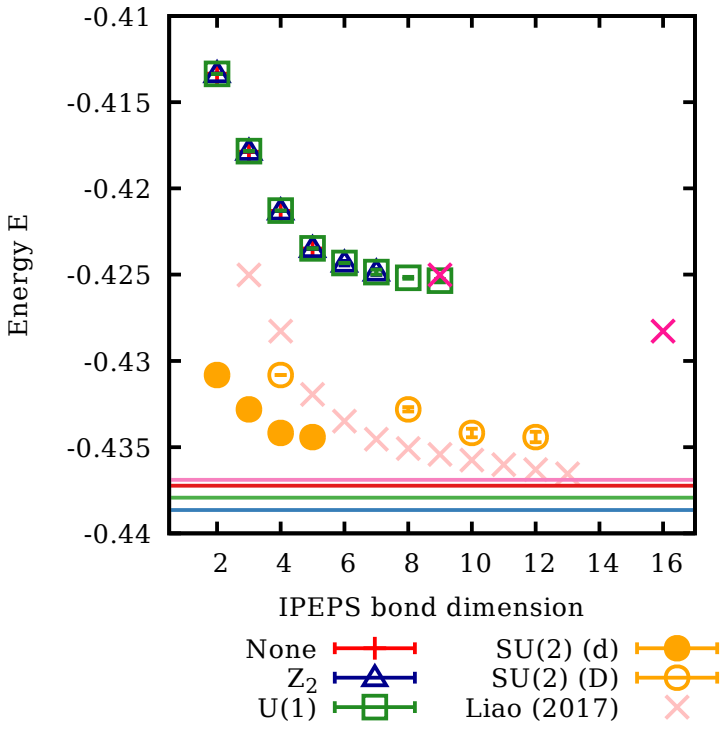

Figure 5: Ground state energy per spin on the Kagomé lattice. The square lattice mapping leads to energies which are considerably worse than the current best estimates $E_{0} \approx 0.437$ (values from [47], [48], [49], [17] shown as lines in top-down order, 9-site PESS from Ref. [48] as pink crosses).

\subsection{The Kagomé lattice}

To benchmark the effect of a $\mathrm{SU}(2)$ spin symmetry which is probably not spontaneously broken in the ground state, we will consider the Heisenberg Hamiltonian on the Kagomé lattice. Our implementation currently only handles square lattices, requiring a mapping of the Kagomé lattice to the square lattice (cf. Fig. 4 and e.g. Ref. [46]). This mapping is exact, the resulting 8-dimensional space of the square lattice sites is not truncated ${ }^{3}$ However, the mapping is not optimal as two links in the Kagomé lattice are represented by just a single link in the square lattice, which then requires an approximately squared bond dimension to represent the same amount of entanglement. This difficulty should affect the calculations with and without symmetries mostly in the same way and hence does not preclude an analysis of the effect of the symmetry implementation here. A smarter approach is to adapt the structure of the tensor network to the Kagomé lattice and potentially introducing additional tensors to aid in convergence 46 48].

Earlier work [47, 48] has found a ground state energy of approximately -0.437 using IPEPS calculations with the simple update mechanism. DMRG calculations on cylinders extrapolated to the $2 \mathrm{D}$ thermodynamic limit resulted in ground state energies per spin of $-0.4386(5)$ [17] if $\mathrm{SU}(2)$ symmetry was conserved and $-0.4379(3)$ without conservation of SU(2) symmetry [49] and subsequently a smaller effective bond dimension.

\footnotetext{
${ }^{3}$ One could, of course, consider such a truncation in future works, e.g. excluding the high-spin states $S=3 / 2$ completely from the calculation.
} 
Fig. 5 shows the results for the energy per spin with and without symmetries for our implementation. The figure also includes reference values as follows: first, solid lines show the extrapolated, final values from Ref. [47], [48], [49] and [17] (in top-down order); the first two are IPEPS calculations whereas the latter two are DMRG calculations on cylinders extrapolated to the thermodynamic limit. Second, light pink crosses reproduce the 9-site PESS data in Ref. [48]. Third, the two bright pink crosses on the right-hand site show the same data, but for an effectively squared bond dimension as Ref. [48 does not use the square lattice mapping. A certain degree of continuity between our data without symmetries and the data from Ref. [48 is visible.

Comparing only our data with and without symmetries, a clear advantage of the implemented $\mathrm{SU}(2)$ symmetry can be observed. Note that this advantage is not solely due to a larger bond dimension, at $D=8$, we have data for both $\mathrm{SU}(2)$ and $\mathrm{U}(1)$-symmetric calculations. Instead, the following effects probably contribute to this advantage: First, enforcing $\mathrm{SU}(2)$ invariance and e.g. avoiding the truncation of a degenerate multiplet in the middle likely improves the convergence of the calculation by avoiding inadequate truncations. Second, the $\mathrm{SU}(2)$ symmetric ansatz state at $d=1$ is often a better initial state, as our Néel-like initial state for $\mathrm{U}(1)$ first has to manually restore the $\mathrm{SU}(2)$ symmetry. In fact, re-running the $\mathrm{SU}(2)$ calculation with a different initial state with a larger spin state on the auxiliary bonds (e.g. $d=1, D=6$ for a $S=5 / 2$ state) leads to considerably worse variational energies at all stages of the calculation. Third, the reduced dense block sizes of the non-abelian calculation certainly increases the numerical stability, as smaller matrices have to be inverted and errors due to the inversion cannot affect the multiplet degeneracy.

This third effect is the most impactful indirect computational advantage of a non-abelian symmetric calculation: At very small bond dimensions, gauge fixing is nearly unnecessary to obtain optimal energies with the full update and the relative advantage of the variational update is small. At larger bond dimensions, the full update without gauge fixing quickly becomes unstable and the relative advantage of the variational update grows. Even when implementing gauge fixing, the full update still plateaus at a relatively high energy which cannot be pushed down further simply by increasing the bond dimension or reducing the Trotter step size. Implementing non-abelian symmetries then transfers the stability of the algorithm for small bond dimensions to much larger bond dimensions and makes it possible to obtain nearly optimal energies.

\subsection{Summary for the computational speed-up}

In this section, we presented results showing that by implementing either abelian or nonabelian symmetries, larger bond dimensions and hence better results are obtainable at comparable computational effort. In the Heisenberg model, $d=10$ is possible when implementing the U(1) symmetry, which approximately doubles the bond dimension compared to our best calculations without any symmetries. As expected, the $\mathbb{Z}_{2}$ symmetry provides a smaller benefit than the U(1) symmetry but still a noticeable speed-up. In the Kagomé model, implementing the $\mathrm{SU}(2)$ symmetry leads to much faster convergence and hence much better results compared to calculations without symmetries.

We must stress that these are "merely" practical advantages: given a sufficiently fast computer, there is no computational benefit in implementing a symmetry into a tensor network. However, we will show in the next section that implementing or not implementing a symmetry provides an additional control which can be used to obtain qualitatively different results. 


\section{$5 \quad$ Diagnosing spontaneous symmetry breaking}

Implementing a symmetry in a system not only allows for a potential computational speed-up, but also provides additional measures to control the calculation.

First, forcing a state to transform uniquely under a symmetry is equivalent to doing the calculation in a canonical instead of grand-canonical ensemble. As a result, it becomes possible to fix total particle number (or average particle number per unit cell) exactly to a desired value. For example in the Kagomé calculation, the states with $\mathrm{SU}(2)$ symmetry are exact eigenstates of the total spin operator with eigenvalue $S \equiv 0$ and the states with U(1) symmetry are exact eigenstates of the total $\hat{S}^{z}$ operator with eigenvalue $S^{z} \equiv 0$. In contrast, the states without any symmetry only have $S^{z} \approx 0$. It then becomes possible to e.g. exactly fix the total particle number per unit cell in the Hubbard model instead of relying on a chemical potential to obtain the desired filling fraction. On the flip side, filling fraction and unit cell size have to be commensurate to provide an integer average particle number per unit cell. This integer average particle number is removed by the "remover" tensor described in Sec. 3.1 and Fig. 1 .

Second, symmetry-preserving tensors can only represent eigenstates of the symmetry. If the symmetry operator and the Hamiltonian commute and are non-degenerate, each eigenstate of the Hamiltonian is also an eigenstate of the symmetry operator and this is no restriction. If, however, the Hamiltonian has a degenerate spectrum, eigenstates of the Hamiltonian are not necessarily eigenstates of the symmetry operator. Then optimising eigenstates of the symmetry operator for the lowest energy does not yield a natural eigenstate of the Hamiltonian but instead some superposition of degenerate eigenstates. Such superpositions have typically larger entanglement, or, if we fix the maximal entanglement, a higher energy than if we allowed breaking of the symmetry in the ansatz.

The increased entanglement of symmetric superpositions can be used to diagnose spontaneous symmetry breaking: If a symmetry is not broken, then it should be possible to implement this symmetry in the tensor network. The resulting state should be an equally good candidate to represent the ground state as a state constructed from unrestricted tensors. As such, at a fixed bond dimension, we expect to find the same variational energy regardless of whether we implement an abelian symmetry or not provided that there are no problems with convergence and the symmetry sectors are chosen in an optimal fashion. Non-abelian symmetries are more tricky, as they increase the effective bond dimension of the state and hence lead to more difficult comparisons. If, on the other hand, a symmetry is spontaneously broken, we expect a better variational energy if we also allow the tensors in our tensor network state to break this symmetry. For lack of spontaneous symmetry breaking in finite and also one-dimensional infinite systems, this is not a problem often encountered. In IPEPS calculations, however, it does become relevant.

The Heisenberg model on the square lattice as discussed before can serve as an example of this effect. The Hamiltonian is:

$$
\hat{H}=\sum_{\langle i, j\rangle} \hat{s}_{i}^{x} s_{j}^{x}+\hat{s}_{i}^{y} \hat{s}_{j}^{y}+|\Delta| \hat{s}_{i}^{z} \hat{s}_{j}^{z}
$$

with now $\Delta$ not fixed at 1 . At $\Delta<1$, the ground state spontaneously breaks the U(1)- $S^{z}$ symmetry and only keeps a canted order. If, for a fixed and finite bond dimension $D$, we then plot the optimal energy obtained over the value of $\Delta$ for different calculations with and 


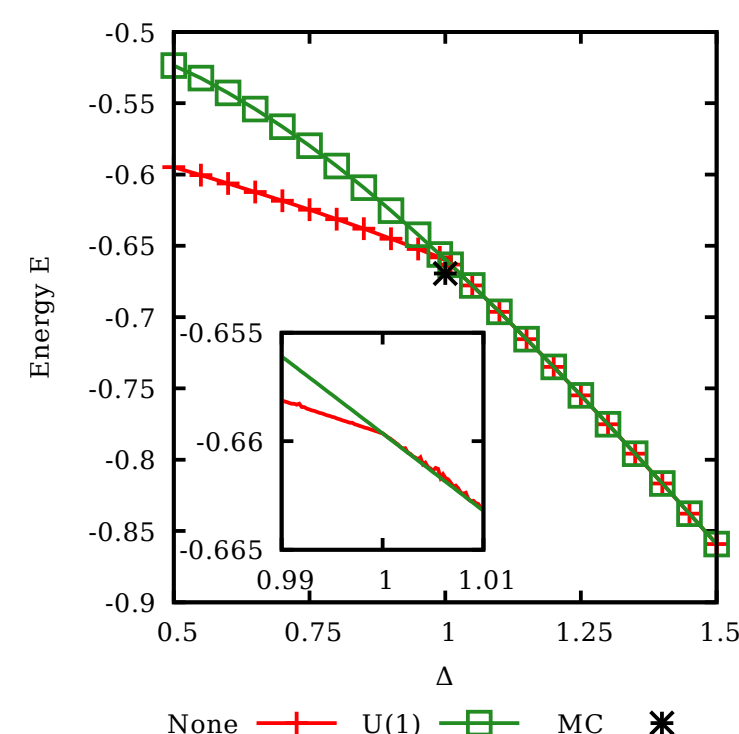

Figure 6: Obtainable ground-state energies in the anisotropic Heisenberg model at $D=$ 2 with and without U(1) symmetry. Inset: Zoom into the central region, $\Delta$ values are placed 0.0025 apart. Lines are guides to the eye. The results start to differ at $\Delta<1$.

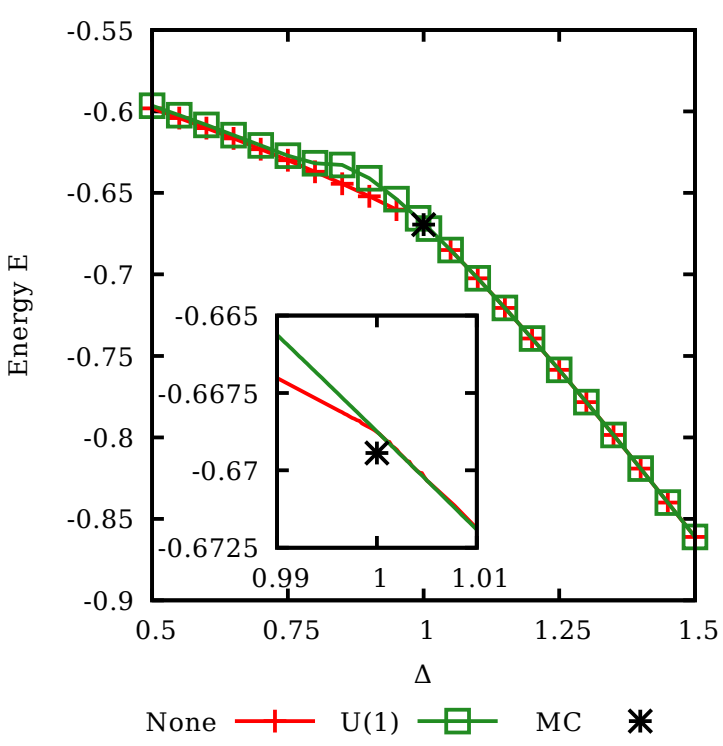

Figure 7: As left, for $D=4$. At small enough $\Delta$, the $\mathrm{U}(1)$ symmetric state becomes competitive again but still has slightly higher energy than the unconstrained state. Monte Carlo reference value at $\Delta=1$ taken from Ref. [45]. Error bars are smaller than symbol size in all cases.

without that symmetry, we can straightforwardly diagnose the transition point, cf. Figs. 6 and 7. Here, we only need to measure the energy of the system - a local observable which is straightforward to evaluate with reliable error bars. More importantly, states are optimised for their energy. The energy is hence typically the observable which behaves in the most stable manner and most closely represents the value obtained in an actual physical state. By comparing the behaviour of the different calculations over the transition point at $\Delta=1$, we can easily conclude that the $\mathrm{U}(1)-S^{z}$ symmetry is spontaneously broken at $\Delta<1$ due to the considerably lower energy obtained from an unconstrained calculation.

Furthermore, while a clear kink at $\Delta=1$ is visible in the energy in calculations without symmetry, this kink is absent and the energy a nearly linear function of $\Delta$ around $\Delta=1$ in the calculation with $\mathrm{U}(1)$ symmetry. The strong bias towards a symmetry-unbroken state completely hides the symmetry-broken phase from the U(1)-symmetric calculation. Hence, by implementing a symmetry in a system, we can not only select a sector in which we want to do our calculation (e.g. $S^{z}=0$ or $N / L=0.5$ ) but also - to some extent - select the symmetries present in the calculated states. Of course, at abitrarily large $D$, it becomes possible for the calculation to hide the symmetry-breaking by a suitable superposition as discussed before. This effect can be seen at $\Delta<0.8$ and $D \geq 4$, where the $\mathrm{U}(1)$-symmetric calculation is again somewhat comparable to the unsymmetric calculation.

If we implement the $\mathbb{Z}_{2}$ symmetry, we disallow unit cell states with $S^{z}= \pm 1$, on the $2 \times 2$ unit cell in a vacuum environment this hence limits us to the two ferromagnetic states 
(which are very highly energetic and don't play a role) and the six $S^{z}=0$ states. With a suitable environment, one could alleviate this problem by combining a $S^{z}=1$ state from the environment with a local $S^{z}=-1$. However, introducing such a state would break the $\mathrm{U}(1)$ symmetry (as it should), which is however preserved by the Hamiltonian we use for the evolution.

Put another way, starting from an antiferromagnetic state $|\psi\rangle$ with $S^{z}=0$, the global ground state is not contained in the Krylov subspace of $\hat{H}$ and $|\psi\rangle$. As such, it is not possible to obtain it by imaginary time evolution alone. Worse, if we initialise the evolution with a symmetry-broken state (of comparatively high energy), the symmetry-broken contribution to this state is very quickly removed by the automatic truncation and hence not available when it would be useful to lower the energy. This problem is not limited to calculations with $\mathbb{Z}_{2}$ symmetry. Also for calculations without any symmetries, it was necessary to select multiple random initial states and run the calculation at $D=2$ multiple times to obtain the correct ground-state energy. Initialising the calculation without symmetries with a Néel state resulted in energies typically very close to those obtained from the calculation with full $U(1)$ symmetry. Only very rarely have we found the error from the truncation to lead to spontaneous symmetry breaking by itself.

Avenues to avoid this problem could be random noise terms added to the state or special "tunneling" Hamiltonians (e.g. $\hat{s}^{+} \hat{s}^{+}+\hat{s}^{-} \hat{s}^{-}$) which are added to the Hamiltonian with a small prefactor and allow for tunneling between different symmetry sectors. The latter approach is already fairly common when solving complicated systems using DMRG. This, however, is outside the scope of the present paper.

\section{Conclusion}

We have shown a considerable computational advantage to be derived from the implementation of symmetries in IPEPS tensor networks. For example implementing a U(1)- $S^{z}$ symmetry in the Heisenberg square lattice model allows for a maximal bond dimension $D=10$ at effort comparable to a calculation without any symmetries at $D=6$. Furthermore, we show that the explicit Clebsch-Gordan Coefficient approach is sufficient to represent $\mathrm{SU}(2)$-symmetric tensors and provides for a large advantage in the Kagomé Heisenberg model. Finally, we provide an example of how implementing a symmetry allows for further control of the calculation by firstly either enabling or disabling the symmetry itself and secondly selecting the desired quantum number sector if the symmetry is enabled.

\section{Acknowledgements}

We are thankful for very helpful discussions with I. P. McCulloch, M. C. Bañuls, J. I. Cirac, L. Vanderstraten, F. Verstraete and N. Schuch.

Funding information Funding through ERC Grant QUENOCOBA, ERC-2016-ADG (Grant no. 742102), is acknowledged. 


\section{References}

[1] U. Schollwöck, The density-matrix renormalization group in the age of matrix product states, Ann. Phys. 326(1), 96 (2011), doi:10.1016/j.aop.2010.09.012.

[2] Y.-Y. Shi, L.-M. Duan and G. Vidal, Classical simulation of quantum manybody systems with a tree tensor network, Phys. Rev. A 74, 022320 (2006), doi:10.1103/PhysRevA.74.022320.

[3] V. Murg, F. Verstraete, O. Legeza and R. M. Noack, Simulating strongly correlated quantum systems with tree tensor networks, Phys. Rev. B 82, 205105 (2010), doi: $10.1103 /$ PhysRevB.82.205105.

[4] L. Tagliacozzo, G. Evenbly and G. Vidal, Simulation of two-dimensional quantum systems using a tree tensor network that exploits the entropic area law, Phys. Rev. B 80, 235127 (2009), doi:10.1103/PhysRevB.80.235127.

[5] M. Gerster, P. Silvi, M. Rizzi, R. Fazio, T. Calarco and S. Montangero, Unconstrained tree tensor network: An adaptive gauge picture for enhanced performance, Phys. Rev. B 90, 125154 (2014), doi:10.1103/PhysRevB.90.125154.

[6] K. Gunst, F. Verstraete, S. Wouters, O. Legeza and D. Van Neck, T3NS: Three-legged tree tensor network states, Journal of Chemical Theory and Computation 14(4), 2026 (2018), doi:10.1021/acs.jctc.8b00098.

[7] D. Bauernfeind, M. Zingl, R. Triebl, M. Aichhorn and H. G. Evertz, Fork tensor-product states: Efficient multiorbital real-time DMFT solver, Phys. Rev. X 7, 031013 (2017), doi: 10.1103/PhysRevX.7.031013.

[8] F. A. Wolf, A. Go, I. P. McCulloch, A. J. Millis and U. Schollwöck, Imaginary-time matrix product state impurity solver for dynamical mean-field theory, Phys. Rev. X 5, 041032 (2015), doi:10.1103/PhysRevX.5.041032.

[9] K. M. Stadler, Z. P. Yin, J. von Delft, G. Kotliar and A. Weichselbaum, Dynamical mean-field theory plus numerical renormalization-group study of spin-orbital separation in a three-band Hund metal, Phys. Rev. Lett. 115, 136401 (2015), doi:10.1103/PhysRevLett.115.136401.

[10] G. Knizia and G. K.-L. Chan, Density matrix embedding: A simple alternative to dynamical mean-field theory, Phys. Rev. Lett. 109, 186404 (2012), doi:10.1103/PhysRevLett.109.186404.

[11] G. Knizia and G. K.-L. Chan, Density matrix embedding: A strong-coupling quantum embedding theory, J. Chem. Theory Comput. 9(3), 1428 (2013), doi:10.1021/ct301044e.

[12] B.-X. Zheng, C.-M. Chung, P. Corboz, G. Ehlers, M.-P. Qin, R. M. Noack, H. Shi, S. R. White, S. Zhang and G. K.-L. Chan, Stripe order in the underdoped region of the two-dimensional Hubbard model, Science 358(6367), 1155 (2017), doi:10.1126/science.aam7127, http://science.sciencemag.org/content/358/6367/ 1155.full.pdf. 
[13] Y. Deng, E. Kozik, N. V. Prokof'ev and B. V. Svistunov, Emergent BCS regime of the two-dimensional fermionic Hubbard model: Ground-state phase diagram, EPL 110(5), 57001 (2015), doi:10.1209/0295-5075/110/57001.

[14] E. Stoudenmire and S. R. White, Studying two-dimensional systems with the density matrix renormalization group, Annu. Rev. Condens. Matter Phys. 3(1), 111 (2012), doi: 10.1146/annurev-conmatphys-020911-125018.

[15] J. P. F. LeBlanc, A. E. Antipov, F. Becca, I. W. Bulik, G. K.-L. Chan, C.-M. Chung, Y. Deng, M. Ferrero, T. M. Henderson, C. A. Jiménez-Hoyos, E. Kozik, X.W. Liu et al., Solutions of the two-dimensional Hubbard model: Benchmarks and results from a wide range of numerical algorithms, Phys. Rev. X 5, 041041 (2015), doi:10.1103/PhysRevX.5.041041.

[16] G. Ehlers, S. R. White and R. M. Noack, Hybrid-space density matrix renormalization group study of the doped two-dimensional Hubbard model, Phys. Rev. B 95, 125125 (2017), doi:10.1103/PhysRevB.95.125125.

[17] S. Depenbrock, I. P. McCulloch and U. Schollwöck, Nature of the spin-liquid ground state of the $S=1 / 2$ Heisenberg model on the Kagome lattice, Phys. Rev. Lett. 109, 067201 (2012), doi:10.1103/PhysRevLett.109.067201.

[18] F. Verstraete and J. I. Cirac, Renormalization algorithms for quantum-many body systems in two and higher dimensions (2004), cond-mat/0407066.

[19] F. Verstraete, V. Murg and J. Cirac, Matrix product states, projected entangled pair states, and variational renormalization group methods for quantum spin systems, Advances in Physics 57(2), 143 (2008), doi:10.1080/14789940801912366, https://doi.org/10.1080/ 14789940801912366 .

[20] J. Jordan, R. Orús, G. Vidal, F. Verstraete and J. I. Cirac, Classical simulation of infinite-size quantum lattice systems in two spatial dimensions, Phys. Rev. Lett. 101, 250602 (2008), doi:10.1103/PhysRevLett.101.250602.

[21] P. Corboz, R. Orús, B. Bauer and G. Vidal, Simulation of strongly correlated fermions in two spatial dimensions with fermionic projected entangled-pair states, Phys. Rev. B 81, 165104 (2010), doi:10.1103/PhysRevB.81.165104.

[22] P. Corboz, J. Jordan and G. Vidal, Simulation of fermionic lattice models in two dimensions with projected entangled-pair states: Next-nearest neighbor hamiltonians, Phys. Rev. B 82, 245119 (2010), doi:10.1103/PhysRevB.82.245119.

[23] P. Corboz, T. M. Rice and M. Troyer, Competing states in the t-J model: Uniform d-wave state versus stripe state: supplemental material, Phys. Rev. Lett. 113, 046402 (2014), doi:10.1103/PhysRevLett.113.046402.

[24] M. Lubasch, J. I. Cirac and M.-C. Bañuls, Algorithms for finite projected entangled pair states, Phys. Rev. B 90, 064425 (2014), doi:10.1103/PhysRevB.90.064425.

[25] H. N. Phien, I. P. McCulloch and G. Vidal, Fast convergence of imaginary time evolution tensor network algorithms by recycling the environment, Phys. Rev. B 91, 115137 (2015), doi:10.1103/PhysRevB.91.115137. 
[26] H. N. Phien, J. A. Bengua, H. D. Tuan, P. Corboz and R. Orús, Infinite projected entangled pair states algorithm improved: Fast full update and gauge fixing, Phys. Rev. B 92, 035142 (2015), doi:10.1103/PhysRevB.92.035142.

[27] L. Vanderstraeten, J. Haegeman, P. Corboz and F. Verstraete, Gradient methods for variational optimization of projected entangled-pair states, Phys. Rev. B 94, 155123 (2016), doi:10.1103/PhysRevB.94.155123.

[28] P. Corboz, Variational optimization with infinite projected entangled-pair states, Phys. Rev. B 94, 035133 (2016), doi:10.1103/PhysRevB.94.035133.

[29] P. Corboz, Improved energy extrapolation with infinite projected entangled-pair states applied to the two-dimensional Hubbard model, Phys. Rev. B 93, 045116 (2016), doi:10.1103/PhysRevB.93.045116.

[30] A. Weichselbaum, Non-abelian symmetries in tensor networks: A quantum symmetry space approach, Ann. Phys. 327(12), 2972 (2012), doi:10.1016/j.aop.2012.07.009.

[31] J. Hauschild, R. S. K. Mong, F. Pollmann, M. Schulz, L. Schoonderwoert, J. Unfried, Y.-C. Tzeng and M. P. Zaletel, Tensor Network Python (TENPY), https://github. com/tenpy/tenpy.

[32] I. P. McCulloch, The Matrix Product Toolkit, https://people.smp.uq.edu.au/ IanMcCulloch/mptoolkit/.

[33] E. M. Stoudenmire, S. R. White and The ITensor Collaboration, ITEnsor $C++$ library, https://itensor.org.

[34] I. P. McCulloch and M. Gulácsi, The non-abelian density matrix renormalization group algorithm, EPL 57(6), 852 (2002), doi:10.1209/epl/i2002-00393-0.

[35] S. Singh and G. Vidal, Tensor network states and algorithms in the presence of a global SU(2) symmetry, Phys. Rev. B 86, 195114 (2012), doi:10.1103/PhysRevB.86.195114.

[36] B. Bauer, P. Corboz, R. Orús and M. Troyer, Implementing global abelian symmetries in projected entangled-pair state algorithms, Phys. Rev. B 83, 125106 (2011), doi:10.1103/PhysRevB.83.125106.

[37] S. Singh, R. N. C. Pfeifer and G. Vidal, Tensor network decompositions in the presence of a global symmetry, Phys. Rev. A 82, 050301 (2010), doi:10.1103/PhysRevA.82.050301.

[38] B. Bruognolo, Tensor network techniques for strongly correlated systems, Ph.D. thesis, LMU Munich (2017).

[39] C. Hubig, F. Lachenmaier, N.-O. Linden, T. Reinhard, L. Stenzel and A. Swoboda, The SYTEN toolkit.

[40] C. Hubig, Symmetry-Protected Tensor Networks, Ph.D. thesis, LMU München (2017).

[41] C. Hubig, I. P. McCulloch, U. Schollwöck and F. A. Wolf, Strictly singlesite DMRG algorithm with subspace expansion, Phys. Rev. B 91, 155115 (2015), doi:10.1103/PhysRevB.91.155115. 
[42] M. Rader and A. M. Läuchli, Finite correlation length scaling in Lorentz-invariant gapless IPEPS wave functions (2018), 1803.08566v1.

[43] A. Hackenbroich, A. Sterdyniak and N. Schuch, Interplay of SU(2), point group and translation symmetry for PEPS: application to a chiral spin liquid (2018), 1805.04531.

[44] C. Hubig, I. P. McCulloch and U. Schollwöck, Generic construction of efficient matrix product operators, Phys. Rev. B 95, 035129 (2017), doi:10.1103/PhysRevB.95.035129.

[45] A. W. Sandvik, Finite-size scaling of the ground-state parameters of the two-dimensional Heisenberg model, Phys. Rev. B 56, 11678 (1997), doi:10.1103/PhysRevB.56.11678.

[46] P. Corboz, K. Penc, F. Mila and A. M. Läuchli, Simplex solids in SU(n) Heisenberg models on the Kagome and checkerboard lattices, Phys. Rev. B 86, 041106 (2012), doi:10.1103/PhysRevB.86.041106.

[47] J.-W. Mei, J.-Y. Chen, H. He and X.-G. Wen, Gapped spin liquid with $\digamma_{2}$ topological order for the Kagome Heisenberg model, Phys. Rev. B 95, 235107 (2017), doi:10.1103/PhysRevB.95.235107.

[48] H. J. Liao, Z. Y. Xie, J. Chen, Z. Y. Liu, H. D. Xie, R. Z. Huang, B. Normand and T. Xiang, Gapless spin-liquid ground state in the $S=1 / 2$ Kagome antiferromagnet, Phys. Rev. Lett. 118, 137202 (2017), doi:10.1103/PhysRevLett.118.137202.

[49] S. Yan, D. A. Huse and S. R. White, Spin-liquid ground state of the $S=1 / 2$ Kagome Heisenberg antiferromagnet, Science 332(6034), 1173 (2011), doi:10.1126/science.1201080. 